TRANSACTIONS OF THE

AMERICAN MATHEMATICAL SOCIETY

Volume 360, Number 2, February 2008, Pages 609-627

S 0002-9947(07)04134-7

Article electronically published on September 21, 2007

\title{
PURE SUBRINGS OF REGULAR RINGS ARE PSEUDO-RATIONAL
}

\author{
HANS SCHOUTENS
}

\begin{abstract}
We prove a generalization conjectured by Aschenbrenner and Schoutens (2003) of the Hochster-Roberts-Boutot-Kawamata Theorem: let $R \rightarrow S$ be a pure homomorphism of equicharacteristic zero Noetherian local rings. If $S$ is regular, then $R$ is pseudo-rational, and if $R$ is moreover $\mathbb{Q}$-Gorenstein, then it is pseudo-log-terminal.
\end{abstract}

\section{INTRODUCTION}

Hochster and Roberts showed in [13, using finite characteristic methods, that quotient singularities in characteristic zero are Cohen-Macaulay. This was improved by Boutot in 2] where he shows, using deep vanishing theorems, that they are rational. More precisely, if $G$ is the complexification of a compact Lie group which acts algebraically on an affine smooth scheme $X$ of finite type over $\mathbb{C}$, then the quotient $X / G$ has rational singularities. In algebraic terms, with $X=\operatorname{Spec} B$, this means that the ring of invariants $A:=B^{G}$ has rational singularities whenever $B$ is regular. (In fact, it suffices that $B$ has at most rational singularities, and there is also a similar result in the analytic category.) When $G$ is finite, Kawamata in [16] showed moreover that $X / G$ has at most log-terminal singularities, and the author showed in [27], using non-standard tight closure, that this remains true for non-finite $G$, provided $X / G$ is moreover $\mathbb{Q}$-Gorenstein (a condition that holds automatically if $G$ is finite).

The goal of the present paper is to extend all these results by removing the finite type condition. However, since the notion of rational singularities is defined in terms of a resolution of singularities, which might not be available in such generality, we need to replace it by the notion of pseudo-rationality.

Main Theorem A. Let $A \rightarrow B$ be a cyclically pure homomorphism of Noetherian rings containing $\mathbb{Q}$. If $B$ is regular, then $A$ is pseudo-rational.

Recall that a homomorphism $A \rightarrow B$ is cyclically pure if $\mathfrak{a}=\mathfrak{a} B \cap A$ for each ideal $\mathfrak{a}$ in $A$; examples are split, pure or faithfully flat homomorphisms. Since the

Received by the editors July 22, 2005.

2000 Mathematics Subject Classification. Primary 14B05, 13H10, 03C20.

Key words and phrases. Tight closure, non-standard Frobenius, rational singularities, Boutot's Theorem, log-terminal singularities.

The author was partially supported by a grant from the National Science Foundation and a PSC-CUNY grant.

(C)2007 American Mathematical Society Reverts to public domain 28 years from publication 
inclusion $B^{G} \subseteq B$ is split (via the so-called Reynolds operator), Boutot's result is therefore just a special case of our first main theorem. Theorem $\mathrm{A}$ was conjectured in [1] and proven for algebras of finite type over an algebraically closed field in 26] using canonical big Cohen-Macaulay algebras. The analogue in prime characteristic was proven by Smith in [28, but unlike most applications of tight closure, this proof did not carry over to characteristic zero, the reason being that cyclic purity is not preserved under reduction modulo $p$. To formulate a corresponding generalization in the $\mathbb{Q}$-Gorenstein case, we need to make a definition. Call a Noetherian local $\mathbb{Q}$ Gorenstein ring $R$ pseudo-log-terminal, if its canonical cover $\tilde{R}$ (see $\$ 7.2$ ) is pseudorational. In particular, if we are in a category of local algebras in which 'pseudorational' is equivalent with 'rational' (e.g., the category of local algebras essentially of finite type over a field), then 'pseudo-log-terminal' is the same as 'log-terminal' by a result of Kawamata (Theorem 7.3). With this terminology, we get the following generalization, conjectured in [1] and proven for algebras of finite type over an algebraically closed field in [27].

Main Theorem B. Let $R \rightarrow S$ be a cyclically pure homomorphism of equicharacteristic zero Noetherian local rings with $S$ regular. If $R$ is $\mathbb{Q}$-Gorenstein, then it is pseudo-log-terminal.

To prove both theorems, we will transform the argument for finitely generated algebras given in 27] by means of the machinery of faithfully flat Lefschetz hulls introduced in [1]. In that paper, we show that given an equicharacteristic zero Noetherian local ring $R$, we can find a faithfully flat local $R$-algebra $\mathfrak{D}(R)$ which is an ultraproduct of rings of prime characteristic (these latter rings are called approximations of $R$, and their ultraproduct is called a Lefschetz hull of $R$ ). These results enabled us in [1] to generalize the alternative constructions of tight closure and big Cohen-Macaulay algebras from the papers 23, 26, 27 to arbitrary equicharacteristic zero Noetherian local rings. Similar applications, although only implicitly using Lefschetz hulls, can be found in [22, 24].

In the present paper, we will concentrate on one variant coming out of this work, to wit, generic tight closure: an element is in the generic tight closure of an ideal if almost all of its approximations belong to the tight closure of the corresponding approximation of the ideal; see $\S 3$ for exact definitions. Theorem $\mathrm{A}$ will follow from the fact that a generically F-rational ring is pseudo-rational (see Theorem 6.2), where we call a ring (generically) F-rational if some ideal generated by a system of parameters is equal to its (generic) tight closure. Smith observes in 28] that Frationality in prime characteristic is equivalent with the top local cohomology of the ring being Frobenius simple. This enables her to prove that an excellent F-rational Noetherian local ring of prime characteristic is pseudo-rational. We will not use this result directly, but rather the method used to prove it. To this end, we also need Lefschetz hulls for finitely generated algebras over a Noetherian local ring, as such rings appear in the Cech complex that calculates the local cohomology. This is carried out in $\oint_{2}$. Therefore, the present proof is entirely self-contained, apart from some material taken from [1].

As for Theorem B we generalize the notion of an ultra-F-regular local ring introduced in 27] as a Noetherian local domain $R$ with the property that for each non-zero $c$, we can find an ultra-Frobenius $\mathbf{F}^{\varepsilon}$ such that the morphism $x \mapsto c \mathbf{F}^{\varepsilon}(x)$ is pure (an ultra-Frobenius is an ultraproduct of powers of Frobenii; see $\$ 2.2$ below). We then show that the property of being ultra-F-regular descends under cyclically 
pure local homomorphisms (Proposition 7.9) and is preserved under finite extensions which are étale in codimension one (Proposition 7.8). Moreover, we show that an ultra-F-regular local ring is pseudo-rational.

\section{Open questions.}

(1) Does the converse of Theorem6.2 also hold, that is to say, is pseudo-rational equivalent with generically F-rational? In [26, Theorem 5.11], I gave a proof of this in the finitely generated case which relies on a deep theorem due to Hara: a local $\mathbb{C}$-algebra $R$ of finite type has rational singularities if and only if it is of F-rational type; see [6].

(2) Does the stronger analogue of Boutot's result also hold, that is to say, can we weaken the assumption in Theorem $A$ that $B$ is only pseudo-rational? In the finitely generated case, a tight closure proof is available if $B$ is moreover Gorenstein ([26, §5.14]), but this again depends on Hara's result.

(3) In 27, using once again Hara's result, it was shown that for $\mathbb{Q}$-Gorenstein local domains of finite type over an algebraically closed field, the notions ultra-F-regular and log-terminal are equivalent. Is ultra-F-regular and pseudo-log-terminal the same for $\mathbb{Q}$-Gorenstein local domains?

(4) Again, we can weaken in the finite type case 27. the assumption that $S$ is regular to the assumption that it is (pseudo-) log-terminal. Does this also hold in general?

(5) For local algebras of finite type over a field of characteristic zero, rational and pseudo-rational are the same notions, and so are log-terminal and pseudo-log-terminal. For which other categories of equicharacteristic zero Noetherian local rings is this the case?

\section{LEFSCHETZ HULLS}

Let $S_{w}$ be a sequence of rings, where $w$ runs over some infinite set endowed with a non-principal ultrafilter. The ultraproduct of this sequence is a ring $S_{\infty}$ given as the homomorphic image of the product $\prod_{w} S_{w}$ modulo the ideal of all sequences which are almost equal to the zero sequence (two sequences $\left(a_{w}\right)$ and $\left(b_{w}\right)$ in the product are said to be almost equal if $a_{w}=b_{w}$ for almost all $w$, that is to say, for all $w$ in some member of the ultrafilter). When we want to emphasize the index, we also denote the ultraproduct $S_{\infty}$ by

$$
\operatorname{ulim}_{w} S_{w}
$$

and similarly, the image of a sequence $\left(a_{w}\right)$ in $S_{\infty}$ is denoted $\operatorname{ulim}_{w} a_{w}$ or simply $a_{\infty}$. In case all rings are equal, say $S_{w}:=S$, their ultraproduct is called an ultrapower of $S$. For more details, see [14, §9.5] or [5], or the brief review in [23, §2].

2.1. Lefschetz hulls. Let $K$ be an uncountable algebraically closed field of characteristic zero. In [1, we associate to every Noetherian local ring $R$ whose residue field is contained in $K$, a faithfully flat Lefschetz hull, that is to say, a faithfully flat extension $R \subseteq \mathfrak{D}(R)$ such that $\mathfrak{D}(R)$ is an ultraproduct of prime characteristic (complete) Noetherian local rings $R_{w}$. Any sequence of prime characteristic complete Noetherian local rings $R_{w}$ whose ultraproduct is equal to $\mathfrak{D}(R)$ is called an approximation of $R$. For the extent to which the assignment $R \mapsto \mathfrak{D}(R)$ is functorial, we refer to the cited paper. All we need in the present paper is that if $R \rightarrow S$ is a local homomorphism of Noetherian local rings whose residue field is contained 
in $K$, then there is a homomorphism $\mathfrak{D}(R) \rightarrow \mathfrak{D}(S)$ making the following diagram commute:

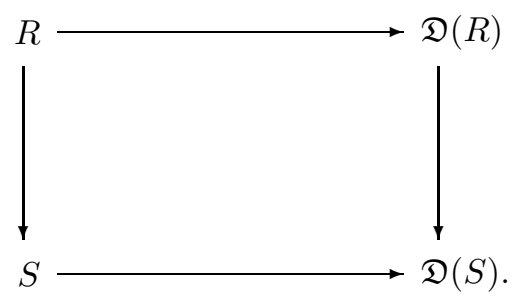

For the remainder of this section, $R$ is an equicharacteristic zero Noetherian local ring, $R_{w}$ an approximation of $R$ and $\mathfrak{D}(R)$ its Lefschetz hull. We always choose $K$ large enough so that it contains all pertinent residue fields, and hence from now on no further reference is made to it. For each $w$, let $\mathbf{F}_{w}$ denote the Frobenius on $R_{w}$, that is to say the homomorphism given by $x \mapsto x^{p(w)}$, where $p(w)$ is the characteristic of $R_{w}$. Given a positive integer $e$, let ${ }^{e} R_{w}$ denote the $R_{w}$-algebra structure on $R_{w}$ given by $\mathbf{F}_{w}^{e}$. It follows that $\mathbf{F}_{w}^{e}: R_{w} \rightarrow{ }^{e} R_{w}$ is $R_{w}$-linear.

2.2. Ultra-Frobenius. A non-standard integer is an element $\varepsilon$ of the ultrapower $\mathbb{Z}_{\infty}$ of $\mathbb{Z}$, that is to say, an ultraproduct of integers $e_{w}$. If almost all $e_{w}$ are positive, then we call $\varepsilon$ positive. For each positive non-standard integer $\varepsilon$, let $\mathbf{F}^{\varepsilon}: R \rightarrow \mathfrak{D}(R)$ be the ultraproduct of the $\mathbf{F}_{w}^{e_{w}}$, that is to say, for $x \in R$ with approximation $x_{w}$, we have

$$
\mathbf{F}^{\varepsilon}(x):=\operatorname{ulim}_{w} \mathbf{F}_{w}^{e_{w}}\left(x_{w}\right) \in \mathfrak{D}(R) .
$$

As in [27, we will call any homomorphism $R \rightarrow \mathfrak{D}(R)$ of the form $\mathbf{F}^{\varepsilon}$ for some $\varepsilon$ an ultra-Frobenius. If $\varepsilon=1$, then the corresponding ultra-Frobenius is just the non-standard Frobenius introduced in [1].

For each positive non-standard integer $\varepsilon$, we may view $\mathfrak{D}(R)$ as an $R$-algebra via the homomorphism $\mathbf{F}^{\varepsilon}$. To denote this algebra structure, we will write ${ }^{\varepsilon} \mathfrak{D}(R)$ (in 27, the alternative notation $\left(\mathbf{F}^{\varepsilon}\right) * \mathfrak{D}(R)$ was used). In other words, the $R$-algebra structure on ${ }^{\varepsilon} \mathfrak{D}(R)$ is given by $x \cdot \alpha:=\mathbf{F}^{\varepsilon}(x) \alpha$, for $x \in R$ and $\alpha \in \mathfrak{D}(R)$.

One of the major drawbacks of the functor $\mathfrak{D}$ is its local nature. In particular, since a localization $R \rightarrow R_{\mathfrak{p}}$ is not a local homomorphism, there is no obvious map from $\mathfrak{D}(R)$ to $\mathfrak{D}\left(R_{\mathfrak{p}}\right)$. Below we will have to deal with localizations of the form $R_{y}$, and hence we need a notion of Lefschetz hull for such (non-local) rings as well.

2.3. $R$-approximations. Let $Y$ be a tuple of indeterminates and let $f \in R[Y]$, say of the form $f=\sum_{\nu \in N} a_{\nu} Y^{\nu}$ with $a_{\nu} \in R$ and $N$ a finite index set. If $a_{\nu w}$ is an approximation of $a_{\nu}$, for each $\nu \in N$, then we call the sequence of polynomials $f_{w}:=\sum_{\nu \in N} a_{\nu w} Y^{\nu}$ an $R$-approximation of $f$.

One checks that any two $R$-approximations of a polynomial $f$ are almost equal. Similarly, if $I:=\left(f_{1}, \ldots, f_{s}\right)$ is an ideal in $R[Y]$ and $f_{i w}$ is an $R$-approximation of $f_{i}$, for each $i$, then we call the sequence $I_{w}:=\left(f_{1 w}, \ldots, f_{s w}\right) R_{w}[Y]$ an $R$ approximation of $I$, and if $S=R[Y] / I$, then we call the sequence $S_{w}:=R_{w}[Y] / I_{w}$ an $R$-approximation of $S$. 
2.4. Relative hulls. If $S$ is a finitely generated $R$-algebra and $S_{w}$ is an $R$-approximation of $S$, then the ultraproduct of the $S_{w}$ is called the (relative) $R$-hull of $S$ and is denoted $\mathfrak{D}_{R}(S)$.

If $R[Z] / J$ is another presentation of $S$ as an $R$-algebra, then we have substitution maps $Y \mapsto \mathbf{a}$ and $Z \mapsto \mathbf{b}$ which induce isomorphisms modulo $I$ and $J$ respectively, where $\mathbf{a}$ and $\mathbf{b}$ are tuples of polynomials in the $Z$ and $Y$-variables respectively. Let $\mathbf{a}_{w}$ and $\mathbf{b}_{w}$ be $R$-approximations of these respective tuples and let $J_{w}$ be an $R$-approximation of $J$. By Łos' Theorem the substitutions $Y \mapsto \mathbf{a}_{w}$ and $Z \mapsto \mathbf{b}_{w}$ induce for almost all $w$ isomorphisms modulo $I_{w}$ and $J_{w}$ respectively. It follows that the ultraproduct of the $R_{w}[Y] / I_{w}$ is isomorphic to the ultraproduct of the $R_{w}[Z] / J_{w}$, showing that $\mathfrak{D}_{R}(S)$ is independent from the particular presentation of $S$ and from the particular choice of $R$-approximations.

Since $\mathfrak{D}_{R}(S)$ is naturally a $\mathfrak{D}(R)$-algebra and since by Łos' Theorem the tuple $Y$ is algebraically independent over $\mathfrak{D}(R)$, we get a natural $\mathfrak{D}(R)[Y]$-algebra structure, whence an $R[Y]$-algebra structure, on $\mathfrak{D}_{R}(S)$. Under the natural homomorphism $R[Y] \rightarrow \mathfrak{D}_{R}(S)$, we get $I \mathfrak{D}_{R}(S)=0$, so that this induces a homomorphism $S \rightarrow$ $\mathfrak{D}_{R}(S)$, endowing $\mathfrak{D}_{R}(S)$ with a canonical $S$-algebra structure. We can now extend the notion of $R$-approximation of an element $a$ or an ideal $\mathfrak{a}$ in a finitely generated $R$-algebra $S$ as follows. Let $S:=R[Y] / I$ and choose a polynomial $f \in R[Y]$ and an ideal $\mathfrak{A}$ in $R[Y]$ so that their images in $S$ are respectively $a$ and $\mathfrak{a}$. Let $f_{w}, \mathfrak{A}_{w}$ and $S_{w}$ be $R$-approximations of $f, \mathfrak{A}$ and $S$ respectively. We call the image $a_{w}$ of $f_{w}$ in $S_{w}$ (respectively, the ideal $\mathfrak{a}_{w}:=\mathfrak{A}_{w} S_{w}$ ) an $R$-approximation of a (respectively, of $\mathfrak{a}$ ). Note that the ultraproduct of the $a_{w}$ (respectively, of the $\mathfrak{a}_{w}$ ) is equal to the image of $a$ in $\mathfrak{D}_{R}(S)$ (respectively, equal to the ideal $\mathfrak{a} \mathfrak{D}_{R}(S)$ ), showing that any two $R$-approximations are almost equal.

If $S \rightarrow T$ is an $R$-algebra homomorphism of finite type, then this extends to an $R$-algebra homomorphism $\mathfrak{D}_{R}(S) \rightarrow \mathfrak{D}_{R}(T)$ giving rise to a commutative diagram

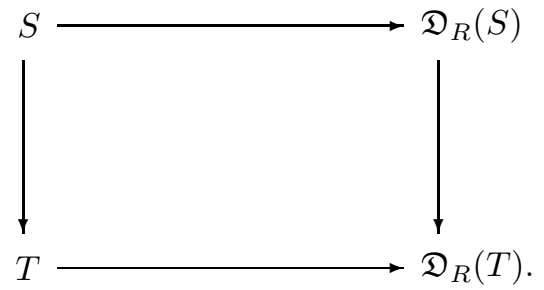

In particular, $\mathfrak{D}_{R}(\cdot)$ is a functor on the category of finitely generated $R$-algebras. The argument is the same as in [23, §3.2.4], and we leave the details to the reader.

\section{Generic tight Closure}

One of the tight closure notions introduced in [1] is generic tight closure. In this section, we review the definition and (re)prove some of its main properties. Throughout this section, $(R, \mathfrak{m})$ will denote an equicharacteristic Noetherian local ring and $\left(R_{w}, \mathfrak{m}_{w}\right)$ one of its approximations. For generalities on (characteristic $p$ ) tight closure, see [15].

3.1. Definition. An element $z \in R$ lies in the generic tight closure of an ideal $\mathfrak{a} \subseteq R$, if almost all $z_{w}$ lie in the tight closure $\mathfrak{a}_{w}^{*}$ of $\mathfrak{a}_{w}$, where $z_{w}$ and $\mathfrak{a}_{w}$ are approximations of $z$ and $\mathfrak{a}$ respectively. 
We denote the generic tight closure of an ideal $\mathfrak{a}$ by $\mathrm{cl}_{\text {gen }}(\mathfrak{a})$. One easily checks that

$$
\operatorname{cl}_{\text {gen }}(\mathfrak{a})=\left(\operatorname{ulim}_{w} \mathfrak{a}_{w}^{*}\right) \cap R
$$

where the contraction is with respect to the canonical embedding $R \rightarrow \mathfrak{D}(R)$. It follows that $\operatorname{cl}_{\text {gen }}(\mathfrak{a})$ is an ideal, containing $\mathfrak{a}$, with the property that $\operatorname{cl}_{\text {gen }}\left(\operatorname{cl}_{\text {gen }}(\mathfrak{a})\right)=$ $\operatorname{cl}_{\text {gen }}(\mathfrak{a})$. We say that an ideal $\mathfrak{a}$ is generically tightly closed if $\mathfrak{a}=\operatorname{cl}_{\text {gen }}(\mathfrak{a})$. The proof of the following easy fact is left to the reader.

3.2. Lemma. If $\mathfrak{a} \subseteq R$ is a generically tightly closed ideal, then so is any colon ideal $\left(\mathfrak{a}:_{R} \mathfrak{b}\right)$, for $\mathfrak{b} \subseteq R$.

3.3. Theorem. If $R$ is regular, then every ideal is generically tightly closed.

Proof. By [1, Theorem 5.2], almost all $R_{w}$ are regular, and hence all ideals in $R_{w}$ are tightly closed by [15, Theorem 1.3]. The assertion then follows from (3) and faithful flatness.

3.4. Theorem (Persistence). If $R \rightarrow S$ is a local homomorphism and $\mathfrak{a}$ an ideal in $R$, then $\operatorname{cl}_{g e n}(\mathfrak{a}) S \subseteq \operatorname{cl}_{g e n}(\mathfrak{a} S)$.

Proof. Immediate from (3) and the fact that persistence holds for each $R_{w} \rightarrow S_{w}$, where $S_{w}$ is an approximation of $S$ (note that $R_{w}$ is complete, so that 15, Theorem 2.3] applies).

3.5. Theorem (Strong Colon Capturing). Let $\left(x_{1}, \ldots, x_{d}\right)$ be part of a system of parameters of $R$. For each $i$, the element $x_{i}$ is a non-zero divisor modulo $\operatorname{cl}_{\text {gen }}\left(\left(x_{1}, \ldots, x_{i-1}\right) R\right)$.

Proof. By downward induction on $i$, it suffices to prove the assertion for $i=d$. To this end, suppose $z x_{d} \in \operatorname{cl}_{\text {gen }}(I)$ with $I:=\left(x_{1}, \ldots, x_{d-1}\right) R$. Let $R_{w}, z_{w}$ and $x_{i w}$ be approximations of $R, z$ and $x_{i}$ respectively and put $I_{w}:=\left(x_{1 w}, \ldots, x_{d-1 w}\right) R_{w}$. By 1, Corollary 5.3], almost all $\left(x_{1 w}, \ldots, x_{d w}\right)$ are part of a system of parameters in $R_{w}$ and $z_{w} x_{d w} \in I_{w}^{*}$. Since each $R_{w}$ is complete, Strong Colon Capturing holds for it, that is to say, $x_{d w}$ is a non-zero divisor modulo $I_{w}^{*}$ (see [15, Theorem 3.1A and Lemma 4.1]). Therefore, $z_{w} \in I_{w}^{*}$, whence $z \in \operatorname{cl}_{\text {gen }}(I)$, as we needed to show.

3.6. Remark. In particular, the usual Colon Capturing holds, that is to say, for each $i$, we have an inclusion $\left(\left(x_{1}, \ldots, x_{i-1}\right) R: x_{i}\right) \subseteq \operatorname{cl}_{\text {gen }}\left(\left(x_{1}, \ldots, x_{i-1}\right) R\right)$. The same proof can also be used to prove the following stronger version (compare with [15, Theorem 9.2]): let $\mathbb{Z}[X] \rightarrow R$ be given by $X_{i} \mapsto x_{i}$ and let $I, J \subseteq \mathbb{Z}[X]$ be ideals. We have an inclusion

$$
\left(\operatorname{cl}_{\text {gen }}(I R): J R\right) \subseteq \operatorname{cl}_{\text {gen }}((I: J) R) .
$$

3.7. Corollary. If $\left(x_{1}, \ldots, x_{d}\right)$ is part of a system of parameters in $R$ and if $\left(x_{1}, \ldots, x_{d}\right) R$ is generically tightly closed, then so is each $\left(x_{1}, \ldots, x_{i}\right) R$, for $i=$ $1, \ldots, d$. In particular, $\left(x_{1}, \ldots, x_{d}\right)$ is a regular sequence.

Proof. The last assertion is clear from Colon Capturing and the first assertion. For the first assertion, it suffices to treat the case $i=d-1$, by downwards induction on $i$. Let $I:=\left(x_{1}, \ldots, x_{d-1}\right) R$ and let $z \in \operatorname{cl}_{\text {gen }}(I)$. Clearly, $z \in \operatorname{cl}_{\text {gen }}\left(I+x_{d} R\right)$ and this latter ideal is just $I+x_{d} R$ by hypothesis. Write $z=a+r x_{d}$, with $a \in I$ and $r \in R$. Therefore, $z-a=r x_{d} \in \operatorname{cl}_{\text {gen }}(I)$. Since $x_{d}$ is a non-zero divisor modulo cl gen $(I)$ 
by Theorem 3.5. we get $r \in \operatorname{cl}_{\text {gen }}(I)$. So, we proved that $\operatorname{cl}_{\text {gen }}(I)=I+x_{d} \operatorname{cl}_{\text {gen }}(I)$. Nakayama's Lemma then yields that $I=\operatorname{cl}_{\text {gen }}(I)$.

3.8. Theorem (Briançon-Skoda). The generic tight closure of an ideal $\mathfrak{a} \subseteq R$ is contained in its integral closure. If $\mathfrak{a}$ is generated by at most $n$ elements, then the integral closure of $\mathfrak{a}^{m+n}$ is contained in $\operatorname{cl}_{\text {gen }}\left(\mathfrak{a}^{m+1}\right)$, for each $m$.

Proof. Let $z \in \operatorname{cl}_{\text {gen }}(\mathfrak{a})$. In order to show that $z$ is integral over $\mathfrak{a}$, it suffices by [1. Lemma 2.3] to show that $z \in \mathfrak{a} V$, for each discrete valuation ring $V$ such that $R \rightarrow V$ is a local homomorphism. Now, persistence (Theorem 3.4) yields that $z$ lies in $\mathrm{cl}_{\text {gen }}(\mathfrak{a} V)$, whence, by Theorem 3.3 , in $\mathfrak{a} V$.

Assume next that $z$ lies in the integral closure of $\mathfrak{a}^{m+n}$, for some $m$ and for $n$ the number of generators of $\mathfrak{a}$. Taking an integral equation witnessing this fact and considering approximations, we see that almost all $z_{w}$ lie in the integral closure of $\mathfrak{a}_{w}^{m+n}$, where $z_{w}$ and $\mathfrak{a}_{w}$ are approximations of $z$ and $\mathfrak{a}$ respectively. By the tight closure Briançon-Skoda Theorem (see for instance [15, Theorem 5.7] for an easy proof), almost all $z_{w}$ lie in the tight closure of $\mathfrak{a}_{w}^{m+1}$ and the result follows.

3.9. Comparison with other tight closure operations. By [1, Theorem 6.21], the generic tight closure of an ideal $\mathfrak{a}$ is contained in its non-standard tight closure, provided $R$ is analytically unramified. This latter condition is imposed to insure the existence of uniform test elements ([1, Proposition 6.20]).

If $R$ is moreover equidimensional and universally catenary, then by [1, Proposition 7.13], the $\mathfrak{B}$-closure $\mathfrak{a} \mathfrak{B}(R) \cap R$ of $\mathfrak{a}$ is contained in its generic tight closure, with equality if $\mathfrak{a}$ is generated by a system of parameters. Here $\mathfrak{B}(R)$ denotes the canonical big Cohen-Macaulay algebra associated to $R$ from [1, §7]. (In the special case that $R$ is a complete domain with algebraically closed residue field, $\mathfrak{B}(R)$ is obtained as the ultraproduct of the absolute integral closures $R_{w}^{+}$.)

\section{Generic F-Rationality}

As before, $R$ is an equicharacteristic zero Noetherian local ring and $R_{w}$ is an approximation of $R$.

4.1. Definition. We say that $R$ is generically F-rational, if there exists a system of parameters $\mathbf{x}$ in $R$ such that $\mathbf{x} R$ is generically tightly closed.

Let us say that $R$ is $\mathfrak{B}$-rational, if there exists a system of parameters $\mathbf{x}$ such that $\mathbf{x} R=\mathbf{x} \mathfrak{B}(R) \cap R$. We will prove below that a ring is generically F-rational if and only if its completion $\widehat{R}$ is. We leave it as an exercise to prove that the same property with ' $\mathfrak{B}$-rational' instead of 'generically F-rational' also holds. Therefore, in view of our discussion in $\$ 3.9$, a ring is generically F-rational if and only if it is $\mathfrak{B}$-rational.

4.2. Theorem. If $R$ is generically F-rational, then it is Cohen-Macaulay.

Proof. Let $\mathrm{x}$ be a system of parameters in $R$ such that $\mathrm{x} R$ is generically tightly closed. By Corollary 3.7, the sequence $\mathbf{x}$ is regular and hence $R$ is Cohen-Macaulay.

4.3. Theorem. If $R$ is generically F-rational, then any ideal generated by part of a system of parameters is generically tightly closed. In particular, $R$ is normal. 
Proof. By Theorem 4.2, we know that $R$ is Cohen-Macaulay. By Corollary 3.7, it suffices to show that any ideal generated by a system of parameters $\left(y_{1}, \ldots, y_{d}\right)$ is generically tightly closed. Reasoning on the top local cohomology, we can find $t \geq 1$ and $a \in R$ such that $\left(y_{1}, \ldots, y_{d}\right) R=\left(\left(x_{1}^{t}, \ldots, x_{d}^{t}\right) R:_{R} a\right)$ (see for instance the proof of [15, Lemma 4.1]). Therefore, if we can show that $\left(x_{1}^{t}, \ldots, x_{d}^{t}\right) R$ is generically tightly closed, then so will $\left(y_{1}, \ldots, y_{d}\right) R$ be by Lemma 3.2 . Hence we have reduced to the case that $y_{i}=x_{i}^{t}$, for some $t \geq 1$.

Let $z \in \operatorname{cl}_{\text {gen }}\left(\left(x_{1}^{t}, \ldots, x_{d}^{t}\right) R\right)$. We need to show that $z \in\left(x_{1}^{t}, \ldots, x_{d}^{t}\right) R$. If some $z x_{i}$ does not lie in $\left(x_{1}^{t}, \ldots, x_{d}^{t}\right) R$, we may replace our original $z$ by this new element. Therefore, we may assume that

$$
z\left(x_{1}, \ldots, x_{d}\right) R \subseteq\left(x_{1}^{t}, \ldots, x_{d}^{t}\right) R .
$$

Since $\left(x_{1}, \ldots, x_{d}\right)$ is $R$-regular, we have

$$
\left(\left(x_{1}^{t}, \ldots, x_{d}^{t}\right) R:\left(x_{1}, \ldots, x_{d}\right) R\right)=\left(x_{1}^{t}, \ldots, x_{d}^{t}, y^{t-1}\right) R,
$$

where $y:=x_{1} \cdots x_{d}$. In summary, we may assume that $z=u y^{t-1}$, for some $u \in R$. By (4), we then get

$$
\begin{aligned}
u \in\left(\operatorname{cl}_{\text {gen }}\left(\left(x_{1}^{t}, \ldots, x_{d}^{t}\right) R\right): y^{t-1}\right) & \subseteq \operatorname{cl}_{\text {gen }}\left(\left(\left(x_{1}^{t}, \ldots, x_{d}^{t}\right) R: y^{t-1}\right)\right) \\
& =\operatorname{cl}_{\text {gen }}\left(\left(x_{1}, \ldots, x_{d}\right) R\right)=\left(x_{1}, \ldots, x_{d}\right) R .
\end{aligned}
$$

Therefore, $z=u y^{t-1}$ lies in $\left(x_{1}^{t}, \ldots, x_{d}^{t}\right) R$, as we wanted to show.

In order to prove that $R$ is normal, it suffices to show that any height one principal ideal $a R$ is integrally closed. Since the integral closure of $a R$ is contained in $\mathrm{cl}_{\text {gen }}(a R)$ by Theorem 3.8 , and since $a$ is part of a system of parameters, the conclusion follows from the first assertion.

4.4. Proposition. A local ring $R$ is generically F-rational if and only if its completion $\widehat{R}$ is. In particular, a generically F-rational ring is analytically unramified.

Proof. Let $\mathrm{x}$ be a system of parameters in $R$ such that $\mathfrak{n}:=\mathrm{x} R$ is generically tightly closed. I claim that $\mathfrak{n} \widehat{R}$ is generically tightly closed, from which it follows that $\widehat{R}$ is generically F-rational. To this end, let $\widehat{z} \in \widehat{R}$ be in the generic tight closure of $\mathfrak{n} \widehat{R}$. Write $\widehat{z}=z+\widehat{w}$ with $z \in R$ and $\widehat{w} \in \mathfrak{n} \widehat{R}$. It follows that $z \in \operatorname{cl}_{\text {gen }}(\mathfrak{n} \widehat{R})$. Let $J$ be the ultraproduct of the $\mathfrak{n}_{w}^{*}$, where $\mathfrak{n}_{w}$ is an approximation of $\mathfrak{n}$. Since $R_{w}$ is also an approximation for $\widehat{R}$, we get $\operatorname{cl}_{\text {gen }}(\mathfrak{n} \widehat{R})=J \cap \widehat{R}$ by (3). Hence $z \in J$, and since $J \cap R=\operatorname{cl}_{\text {gen }}(\mathfrak{n})=\mathfrak{n}$, we get $\widehat{z}=z+\widehat{w} \in \mathfrak{n} \widehat{R}$.

Conversely, suppose $\widehat{R}$ is generically F-rational. Let $\mathbf{x}$ be a system of parameters in $R$. Let $a$ be in the generic tight closure of $\mathrm{x} R$, whence by persistence (Theorem (3.4), in the generic tight closure of $\mathbf{x} \widehat{R}$. Since $\mathbf{x}$ is a system of parameters in $\widehat{R}$, the ideal $\mathbf{x} \widehat{R}$ is generically tightly closed by Theorem 4.3. Hence, $a \in \mathbf{x} \widehat{R}$, and therefore, by faithful flatness, $a \in \mathrm{x} R$, proving that $R$ is generically F-rational.

To prove the last assertion, assume $R$ is generically F-rational. Hence so is $\widehat{R}$ by what we just proved. Therefore, $\widehat{R}$ is normal by Theorem 4.3, whence a domain, showing that $R$ is analytically unramified.

4.5. Corollary. If $R$ is generically F-rational, then almost all $R_{w}$ are Cohen-Macaulay and normal.

Proof. Since $\widehat{R}$ and $R$ have the same approximations, we may assume by Proposition 4.4 that $R$ is complete. Theorems 4.2 and 4.3 yield that $R$ is normal and 
Cohen-Macaulay. By [1, Theorem 5.2], almost all $R_{w}$ are Cohen-Macaulay. Since $R$ satisfies Serre's condition $\left(R_{1}\right)$, so do almost all $R_{w}$ by [1, Theorem 5.6]. Together with the fact that almost all $R_{w}$ are Cohen-Macaulay, we get from Serre's criterion for normality (see for instance [19, Theorem 23.8]) that almost all $R_{w}$ are normal.

4.6. Proposition. If almost all $R_{w}$ are F-rational, then $R$ is generically F-rational. The converse holds if $R$ is moreover Gorenstein.

Proof. Let $\mathbf{x}$ be a system of parameters in $R$, with approximation $\mathbf{x}_{w}$, and let $z$ be in the generic tight closure of $\mathbf{x} R$. By [1, Corollary 5.4], almost all $\mathbf{x}_{w}$ are systems of parameters in $R_{w}$. Hence, by definition of F-rationality, $\mathbf{x}_{w} R_{w}$ is tightly closed. Therefore, if $z_{w}$ is an approximation of $z$, then $z_{w} \in \mathbf{x}_{w} R_{w}$. Taking ultraproducts, we see that $z$ lies in $\mathbf{x} \mathfrak{D}(R)$ and hence by faithful flatness, in $\mathbf{x} R$, showing that $R$ is generically F-rational.

Suppose next that $R$ is Gorenstein and generically F-rational. Towards a contradiction, assume almost each $R_{w}$ is not F-rational. If $J$ is the ultraproduct of the $\left(\mathbf{x}_{w} R_{w}\right)^{*}$, then this means that $\mathbf{x} \mathfrak{D}(R) \varsubsetneqq J$. On the other hand, by (3) and our assumption, $J \cap R=\mathrm{x} R$. Put $S:=R / \mathbf{x} R$. By [1, $\S 4.9]$, we have an isomorphism $\mathfrak{D}(S) \cong \mathfrak{D}(R) / \mathbf{x} \mathfrak{D}(R)$ and $\mathfrak{D}(S)$ is an ultrapower of $S \otimes_{k} K$, where $k$ is the residue field of $R$ and $K$ the algebraically closed field used in the definition of Lefschetz hull. Since $S$ is Gorenstein, so is $S \otimes_{k} K$, whence also $\mathfrak{D}(S)$, since the Gorenstein property is first order definable (see for instance 21]). Let $a \in R$ be such that its image in $S$ generates the socle of this ring. By faithful flatness, $a$ is a non-zero element in the socle of $\mathfrak{D}(S)$, whence must generate it. Since $J \mathfrak{D}(S) \neq 0$, we must have $a \in J$ whence $a \in J \cap R=\mathrm{x} R$, a contradiction.

4.7. Remark. Note that by Smith's result [28, Theorem 3.1], an F-rational excellent local ring is pseudo-rational; the converse holds by 6 . It follows that if almost all approximations of $R$ are pseudo-rational, then $R$ is generically F-rational, whence pseudo-rational by Theorem 6.2 below. I do not know whether the converse also holds.

Let us call $R$ weakly generically F-regular, if each ideal $\mathfrak{a} \subseteq R$ is generically tightly closed. By Theorem 3.3. any regular local ring is weakly generically Fregular. By a similar argument as in the proof of Proposition 4.4 one can show that $R$ is weakly generically F-regular if and only if its completion is. If a ring is weakly generically F-regular, then it is generically F-rational; the converse is true for Gorenstein rings, as we now prove.

4.8. Theorem. If $R$ is Gorenstein and generically F-rational, then it is weakly generically F-regular.

Proof. Given an arbitrary ideal $\mathfrak{a} \subseteq R$, we need to show that $\mathfrak{a}=\operatorname{cl}_{\text {gen }}(\mathfrak{a})$. Since $\mathfrak{a}$ is the intersection of $\mathfrak{m}$-primary ideals, we easily reduce to the case that $\mathfrak{a}$ is $\mathfrak{m}$-primary. Choose a system of parameters $\mathrm{x}$ such that $\mathrm{x} R \subseteq \mathfrak{a}$. By Theorem 4.3, the ideal $\mathrm{x} R$ is generically tightly closed. Since $R$ is Gorenstein,

$$
\mathfrak{a}=(\mathbf{x} R:(\mathbf{x} R: \mathfrak{a}))
$$

which is a generically tightly closed ideal by Lemma 3.2 
4.9. Proposition. Let $R \rightarrow S$ be a cyclically pure, local homomorphism between equicharacteristic zero Noetherian local rings. If $S$ is weakly generically F-regular, then so is $R$.

Proof. Let $z \in \mathrm{cl}_{\text {gen }}(\mathfrak{a})$, for $\mathfrak{a}$ an ideal in $R$. By Theorem 3.4 the image of $z$ in $S$ lies in the generic tight closure of $\mathfrak{a} S$, which by assumption is just $\mathfrak{a} S$. Hence $z \in \mathfrak{a} S \cap R=\mathfrak{a}$.

4.10. Remark. It is well-known that the localization of an F-rational ring is again F-rational (see [15, Theorem 4.2]; the same property for weakly F-regular rings, though, is still open). However, since Lefschetz hulls are not compatible with localization, I do not know whether the localization of a generically F-rational ring is again generically F-rational.

The next Briançon-Skoda type theorem was proven first in [18 for pseudorational local rings. Since we will show in the next section that a generically F-rational local ring is pseudo-rational, this version generalizes their result.

4.11. Theorem. If $R$ is a d-dimensional generically F-rational local ring, then the integral closure of $\mathfrak{a}^{m+d}$ is contained in $\mathfrak{a}^{m+1}$, for all $m$ and all ideals $\mathfrak{a} \subseteq R$.

Proof. We follow the argument in [26, Theorem 6.4], where the special case that $R$ is of finite type over an algebraically closed field is proven. Let $a$ be an element of the integral closure of $\mathfrak{a}^{m+d}$. Assume first that $\mathfrak{a}$ is generated by a system of parameters. Therefore, $a$ lies in $\operatorname{cl}_{\text {gen }}\left(\mathfrak{a}^{m+1}\right)$, by Theorem 3.8, whence in $\mathfrak{a}^{m+1}$, by Lemma 4.12 below. This proves the assertion for parameter ideals. Assume next that $\mathfrak{a}$ is merely $\mathfrak{m}$-primary, where $\mathfrak{m}$ is the maximal ideal of $R$. In that case, $\mathfrak{a}$ admits a reduction $I$ generated by a system of parameters. Since $I^{m+d}$ is then a reduction of $\mathfrak{a}^{m+d}$, we get that $a$ lies in the integral closure of $I^{m+d}$, whence in $I^{m+1}$, by the first case, and, therefore, ultimately in $\mathfrak{a}^{m+1}$, also establishing this case. For arbitrary $\mathfrak{a}$, write $\mathfrak{a}$ as the intersection of all $\mathfrak{a}+\mathfrak{m}^{n}$ and use the previous case.

4.12. Lemma. If $(R, \mathfrak{m})$ is a generically F-rational local ring, $\left(x_{1}, \ldots, x_{d}\right)$ a system of parameters and $J$ an $\mathfrak{m}$-primary ideal generated by monomials in the $x_{i}$, then $J$ is generically tightly closed.

Proof. By [4], we can write $J$ as the intersection of ideals of the form $\left(x_{1}^{e_{1}}, \ldots, x_{d}^{e_{d}}\right) R$, for some non-zero $e_{i}$. Each such ideal is generically tightly closed by Theorem 4.3 . whence so is $J$.

\section{LOCAL COHOMOLOGY}

Before we turn to pseudo-rationality, we must say something about local and sheaf cohomology and their respective ultraproducts. For our purposes, local cohomology is most conveniently approached via Čech cohomology, which we quickly review. Let $\mathfrak{a}$ be an ideal in a Noetherian ring $S$ and choose a tuple $\mathbf{x}:=\left(x_{1}, \ldots, x_{d}\right)$ so that $\mathfrak{a}$ and $\mathbf{x} S$ have the same radical. For each $i \leq d$, define

$$
\mathrm{C}^{i}(\mathbf{x} ; S):=\bigoplus_{1 \leq l_{1}<l_{2}<\cdots<l_{i} \leq d} S_{x_{l_{1}} x_{l_{2}} \cdots x_{l_{i}}}
$$

(with the convention that $\left.\mathrm{C}^{0}(\mathbf{x} ; S)=S\right)$. The $\mathrm{C}^{i}(\mathbf{x} ; S)$ are the modules appearing in a complex $\mathrm{C}^{\bullet}(\mathbf{x} ; S)$, called the algebraic $\check{C}$ ech complex with respect to $\mathbf{x}$, where 
the differential $\mathrm{C}^{i}(\mathbf{x} ; S) \rightarrow \mathrm{C}^{i+1}(\mathbf{x} ; S)$ is given by the inclusion maps among the localizations, with the choice of an appropriate sign to make $\mathrm{C}^{\bullet}(\mathbf{x} ; S)$ a complex (see [3, §3.5] for more details). The cohomology of this complex is called the local cohomology of $S$ with respect to $\mathfrak{a}$ and is denoted $\mathrm{H}_{\mathfrak{a}}^{\bullet}(S)$. One shows that $\mathrm{H}_{\mathfrak{a}}^{\bullet}(S)$ only depends on the radical of $\mathfrak{a}$ and, in particular, is independent from the choice of $d$-tuple $\mathbf{x}$. We will be mainly interested in the top cohomology group $\mathrm{H}_{\mathfrak{a}}^{d}(S)$, and we use the following notation. Since $\mathrm{H}_{\mathfrak{a}}^{d}(S)$ is a homomorphic image of $\mathrm{C}^{d}(\mathbf{x} ; S)=S_{x_{1} \ldots x_{d}}$, an arbitrary element is the image of a fraction $\frac{a}{\left(x_{1} \ldots x_{d}\right)^{n}}$, and we will denote this image by $\left[\frac{a}{\left(x_{1} \ldots x_{d}\right)^{n}}\right]_{S}$.

Local cohomology and sheaf cohomology. Let $Y$ be a scheme and $Z$ a closed subset of $Y$. The collection of those global sections in $\mathrm{H}^{0}\left(Y, \mathcal{O}_{Y}\right)$ whose support is contained in $Z$ is denoted $\mathrm{H}_{Z}^{0}(Y)$ and is called the global sections with support in $Z$. The derived functors $\mathrm{H}_{Z}^{i}(Y)$ of the left-exact functor $\mathrm{H}_{Z}^{0}$ are called the cohomology with support in $Z$. The cohomology groups with support are connected to the usual sheaf cohomology via an exact sequence

$$
\cdots \rightarrow \mathrm{H}^{i-1}\left(Y, \mathcal{O}_{Y}\right) \stackrel{\rho^{i-1}}{\longrightarrow} \mathrm{H}^{i-1}\left(Y-Z, \mathcal{O}_{Y-Z}\right) \stackrel{\partial^{i}}{\longrightarrow} \mathrm{H}_{Z}^{i}(Y) \rightarrow \mathrm{H}^{i}\left(Y, \mathcal{O}_{Y}\right) \rightarrow \ldots
$$

where $\partial^{i}$ are the connecting morphisms (see for instance [7, Corollary 1.9]).

For (quasi-)projective schemes, we also have a relationship between local cohomology and sheaf cohomology as follows. Let $R$ be a Noetherian ring. A standard graded $R$-algebra is a Noetherian graded ring

$$
S=\bigoplus_{n \geq 0}[S]_{n}
$$

such that $R=[S]_{0}$ and $S$ is (finitely) generated as an $R$-algebra by $[S]_{1}$. The irrelevant ideal of $S$ will be denoted by $S^{+}:=\bigoplus_{n>0}[S]_{n}$. Let $Y:=\operatorname{Proj} S$ be the projective scheme over Spec $R$ defined by $S$ and let $Z$ be a closed subset of $Y$, defined by some homogeneous ideal $\mathfrak{a} \subseteq S$. For each $i \geq 2$, we have

$$
\mathrm{H}^{i-1}\left(Y-Z, \mathcal{O}_{Y-Z}\right) \cong\left[\mathrm{H}_{\mathfrak{a}}^{i}(S)\right]_{0} .
$$

Local ultracohomology. For the remainder of this section, $R$ is an equicharacteristic zero Noetherian local ring and $S$ is a finitely generated $R$-algebra. Let $\mathfrak{a}$ be an ideal in $S$ and let $\mathbf{x}$ be a $d$-tuple in $S$ such that $\mathfrak{a}$ and $\mathbf{x} S$ have the same radical. Note that each module in the algebraic Čech complex $\mathrm{C}^{\bullet}(\mathbf{x} ; S)$ is a finitely generated $R$-algebra, whence admits an $R$-hull. The non-standard algebraic $\check{C}$ ech complex $\mathrm{C}_{\infty}^{\bullet}(\mathbf{x} ; S)$ over $S$ with respect to $\mathbf{x}$ is by definition the complex whose $i$ th module is $\mathfrak{D}_{R}\left(\mathrm{C}^{i}(\mathbf{x} ; S)\right)$ and for which the differentials are induced by the differentials on $\mathrm{C}^{\bullet}(\mathbf{x} ; S)$. The local ultracohomology of $S$ with respect to $\mathfrak{a}$ is by definition the cohomology of the non-standard algebraic Čech complex $\mathrm{C}_{\infty}^{\bullet}(\mathbf{x} ; S)$ and is denoted $\mathrm{UH}_{\mathfrak{a}}^{\bullet}(S)$.

Without proof, we state that $\mathrm{UH}_{\mathfrak{a}}^{\bullet}(S)$ is independent from the choice of a $d$-tuple $\mathbf{x}$. By (2), the canonical homomorphisms $\mathrm{C}^{i}(\mathbf{x} ; S) \rightarrow \mathfrak{D}_{R}\left(\mathrm{C}^{i}(\mathbf{x} ; S)\right)$ give rise to a map of complexes $\mathrm{C}^{\bullet}(\mathbf{x} ; S) \rightarrow \mathrm{C}_{\infty}^{\bullet}(\mathbf{x} ; S)$, and hence for each $i \leq d$, we get a natural morphism

$$
j_{\mathfrak{a}}^{i}: \mathrm{H}_{\mathfrak{a}}^{i}(S) \rightarrow \mathrm{UH}_{\mathfrak{a}}^{i}(S) .
$$

Let $S_{w}, \mathfrak{a}_{w}$ and $\mathbf{x}_{w}$ be $R$-approximations of $S, \mathfrak{a}$ and $\mathbf{x}$ respectively. Since we can calculate the local cohomology $\mathrm{H}_{\mathfrak{a}_{w}}^{\bullet}\left(S_{w}\right)$ with aid of the algebraic Cech complex of 
$\mathbf{x}_{w}$ and since taking ultraproducts commutes with cohomology, we get

$$
\mathrm{UH}_{\mathfrak{a}}^{i}(S) \cong \operatorname{ulim}_{w} \mathrm{H}_{\mathfrak{a}_{w}}^{i}\left(S_{w}\right)
$$

for each $i$. In particular, if $\varphi: S \rightarrow T$ is an $R$-algebra homomorphism of finite type, then the diagram

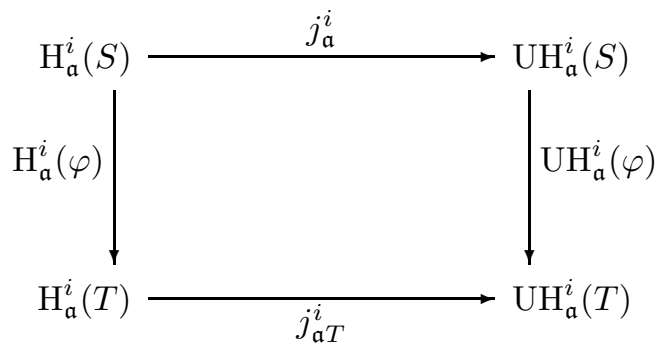

commutes for each $i$, where the vertical arrows are the natural maps.

Sheaf ultracohomology. Assume moreover that $S$ is a standard graded $R$-algebra and $\mathfrak{a}$ is homogeneous. By an argument similar to the one in [27, §2.9], almost all $S_{w}$ are standard graded $R_{w}$-algebras and almost all $\mathfrak{a}_{w}$ are homogeneous. For each non-standard integer $n_{\infty}:=\operatorname{ulim}_{w} n_{w}$ we define the degree $n_{\infty}$ part of $\mathfrak{D}_{R}(S)$ as

$$
\left[\mathfrak{D}_{R}(S)\right]_{n_{\infty}}:=\operatorname{ulim}_{w}\left[S_{w}\right]_{n_{w}} .
$$

If we apply this to each term in the algebraic Čech complex for $\mathfrak{a}$ and take cohomology, we get the degree $n_{\infty}$ part of the non-standard local cohomology groups $\mathrm{UH}_{\mathfrak{a}}^{i}(S)$, and by (7) this is also equal to the ultraproduct of the degree $n_{w}$ parts of the local cohomology of the approximations. In view of isomorphism (6), we define for $i=2, \ldots, d$ the sheaf ultracohomology of $Y-Z$ as

$$
\mathrm{UH}^{i-1}\left(Y-Z, \mathcal{O}_{Y-Z}\right):=\left[\mathrm{UH}_{\mathfrak{a}}^{i}(S)\right]_{0} .
$$

It follows from (6) and (7) that

$$
\mathrm{UH}^{i-1}\left(Y-Z, \mathcal{O}_{Y-Z}\right)=\operatorname{ulim}_{w} \mathrm{H}^{i-1}\left(Y_{w}-Z_{w}, \mathcal{O}_{Y_{w}-Z_{w}}\right),
$$

where $Z_{w}:=\mathrm{V}\left(\mathfrak{a}_{w}\right)$. The natural map $j_{\mathfrak{a}}^{i}: \mathrm{H}_{\mathfrak{a}}^{i}(S) \rightarrow \mathrm{UH}_{\mathfrak{a}}^{i}(S)$ induces in degree zero a map

$$
u_{Y-Z}^{i-1}: \mathrm{H}^{i-1}\left(Y-Z, \mathcal{O}_{Y-Z}\right) \rightarrow \mathrm{UH}^{i-1}\left(Y-Z, \mathcal{O}_{Y-Z}\right) .
$$

The restriction maps induce a diagram

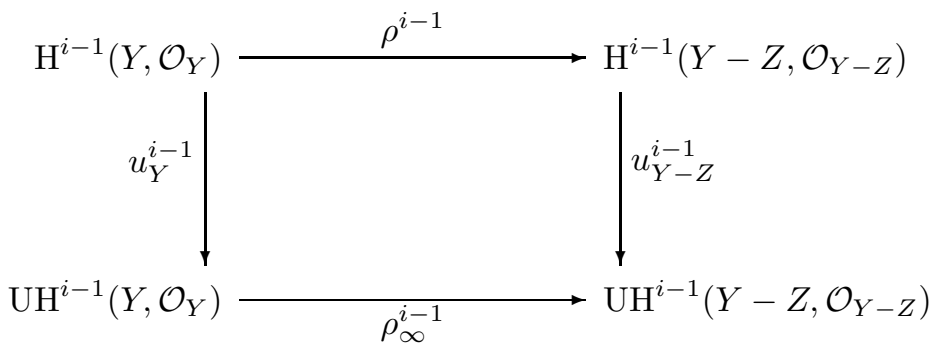

where $\rho_{\infty}^{i-1}$ is the ultraproduct of the restriction maps

$$
\rho_{w}^{i-1}: \mathrm{H}^{i-1}\left(Y_{w}, \mathcal{O}_{Y_{w}}\right) \rightarrow \mathrm{H}^{i-1}\left(Y_{w}-Z_{w}, \mathcal{O}_{Y_{w}-Z_{w}}\right) .
$$


Making the appropriate identifications between local cohomology and sheaf cohomology given by (6), diagram (9) is the degree zero part of

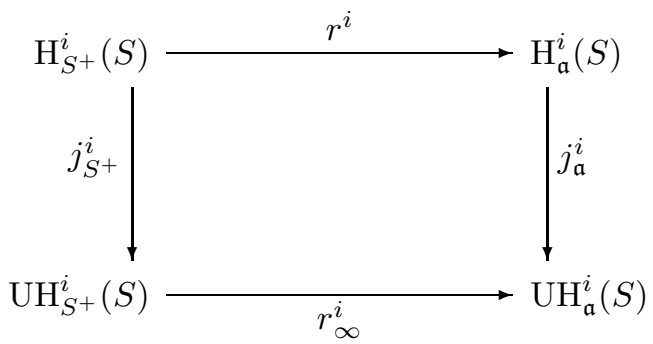

where $r_{\infty}^{i}$ is the ultraproduct of the natural maps

$$
r_{w}^{i}: \mathrm{H}_{S_{w}^{+}}^{i}\left(S_{w}\right) \rightarrow \mathrm{H}_{\mathfrak{a}_{w}}^{i}\left(S_{w}\right) .
$$

It is easy to check that (10) commutes, whence so does (9).

\section{Pseudo-Rationality}

The notion of pseudo-rationality was introduced by Lipman and Teissier to extend the notion of rational singularities to a situation where there is not necessarily a resolution of singularities available.

6.1. Pseudo-rationality. A Noetherian local ring $(R, \mathfrak{m})$ is called pseudo-rational, if it is analytically unramified, normal, Cohen-Macaulay and for any projective birational map $f: Y \rightarrow \operatorname{Spec} R$ with $Y$ normal, the canonical epimorphism between the top cohomology groups $\delta: H_{\mathfrak{m}}^{d}(R) \rightarrow H_{Z}^{d}(Y)$ is injective, where $Z$ is the closed fiber $f^{-1}(\mathfrak{m})$ and $d$ the dimension of $R$ (see (11) below for the definition of $\delta$ ). Moreover, if Spec $R$ admits a desingularization $Y \rightarrow \operatorname{Spec} R$, then it suffices to check the above condition for just this one $Y$ (see [18, §2, Remark (a) and Example (b)]). From this, one can show using Matlis duality, that if $R$ is essentially of finite type over a field of characteristic zero, then $R$ is pseudo-rational if and only if it has rational singularities. A Noetherian ring $A$ is called pseudo-rational, if $A_{\mathfrak{p}}$ is pseudo-rational for every prime ideal $\mathfrak{p}$ in $A$.

The key ingredient in proving Theorems $\mathrm{A}$ and $\mathrm{B}$ is the following result linking generic tight closure with pseudo-rationality, analogous to Smith's characterization 28] in prime characteristic.

6.2. Theorem. If an equicharacteristic zero Noetherian local ring $R$ is generically F-rational, then it is pseudo-rational.

Proof. By Theorems 4.2 and 4.3 and Proposition 4.4 we know that $R$ is analytically unramified, Cohen-Macaulay and normal. Let $X:=\operatorname{Spec} R$ and let $f: Y=$ $\operatorname{Proj} S \rightarrow X$ be a projective birational map with $Y$ normal. In particular, $S$ is a standard graded $R$-algebra. Let $i: R \rightarrow S$ be the embedding identifying $R$ with $[S]_{0}$, let $\mathfrak{m}$ be the maximal ideal of $R$ and let $Z:=\mathrm{V}(\mathfrak{m} S)$ be the closed fiber of $f$. The image of the canonical map $\mathrm{H}_{\mathfrak{m}}^{d}(i): \mathrm{H}_{\mathfrak{m}}^{d}(R) \rightarrow \mathrm{H}_{\mathfrak{m} S}^{d}(S)$ lies entirely in degree zero, whence in view of (6), induces a morphism $\gamma^{d}: \mathrm{H}_{\mathfrak{m}}^{d}(R) \rightarrow \mathrm{H}^{d-1}\left(Y-Z, \mathcal{O}_{Y-Z}\right)$. 
Combining this with the tail of the exact sequence (5) and with (9) gives a commutative diagram

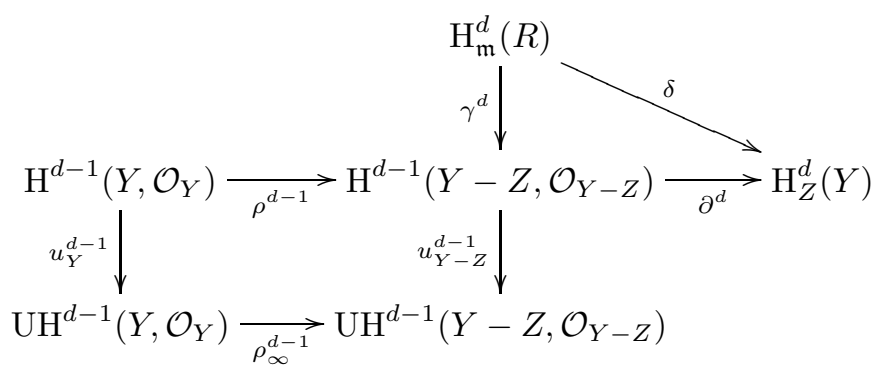

in which the middle row is exact.

Let $\mathbf{x}$ be a system of parameters in $R$ such that $\mathbf{x} R$ is generically tightly closed. Note that the algebraic Cech complex of $\mathrm{x}$ over $R$ (respectively, over $S$ ) calculates the local cohomology of $\mathfrak{m}$ (respectively, of $\mathfrak{m} S$ ). We need to show that the kernel of $\delta$ is zero, hence suppose the contrary. In particular, it must contain a non-zero element of the form $\left[\frac{a}{y}\right]_{R}$, with $a \in R$ and where $y$ is the product of the entries in x. From the exactness of (11), we see that $\delta\left(\left[\frac{a}{y}\right]_{R}\right)=0$ means that $\gamma^{d}\left(\left[\frac{a}{y}\right]_{R}\right)$ lies in the image of $\rho^{d-1}$. Under the isomorphism $\mathrm{H}^{d-1}\left(Y-Z, \mathcal{O}_{Y-Z}\right) \cong\left[\mathrm{H}_{\mathfrak{m} S}^{d}(S)\right]_{0}$ from ([6), we may identify $\gamma^{d}\left(\left[\frac{a}{y}\right]_{R}\right)$ with $\left[\frac{a}{y}\right]_{S}$. Since the square in (11) commutes, $u_{Y-Z}^{d-1}\left(\left[\frac{a}{y}\right]_{S}\right)$ lies in the image of $\rho_{\infty}^{d-1}$.

Let $\left(R_{w}, \mathfrak{m}_{w}\right)$ be an approximation of $(R, \mathfrak{m})$. By Corollary [4.5, almost all $R_{w}$ are Cohen-Macaulay and normal, whence in particular domains. Let $S_{w}$ be an $R$-approximation of $S$, put $X_{w}:=\operatorname{Spec}\left(R_{w}\right)$ and $Y_{w}:=\operatorname{Proj}\left(S_{w}\right)$, and let $Z_{w}:=$ $\mathrm{V}\left(\mathfrak{m}_{w} S_{w}\right)$ be the closed fiber of $Y_{w} \rightarrow X_{w}$. Let $a_{w}$ and $\mathbf{x}_{w}$ be approximations of $a$ and $\mathbf{x}$ respectively, and put $y_{w}$ equal to the product of all the entries in $\mathbf{x}_{w}$. By definition, $u_{Y-Z}^{d-1}\left(\left[\frac{a}{y}\right]_{S}\right)$ is the ultraproduct of the $\left[\frac{a_{w}}{y_{w}}\right]_{S_{w}}$. Hence by Los' Theorem, almost each $\left[\frac{a_{w}}{y_{w}}\right]_{S_{w}}$ lies in the image of

$$
\rho_{w}^{d-1}: \mathrm{H}^{d-1}\left(Y_{w}, \mathcal{O}_{Y_{w}}\right) \rightarrow \mathrm{H}^{d-1}\left(Y_{w}-Z_{w}, \mathcal{O}_{Y_{w}-Z_{w}}\right)
$$

since $\rho_{\infty}^{d-1}$ is the ultraproduct of the $\rho_{w}^{d-1}$. By the same argument as above, we have for each $w$, an exact diagram

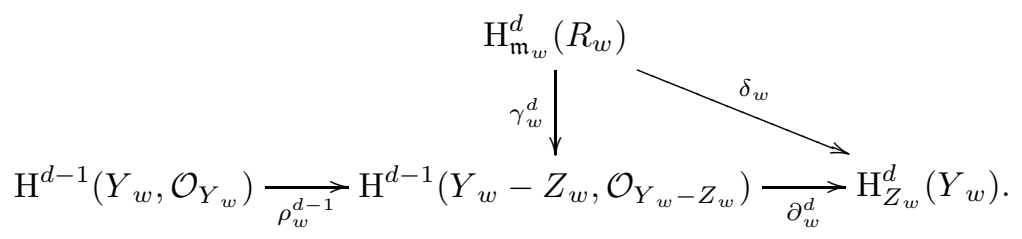

By reversing the above arguments, this diagram then shows that almost each $\left[\frac{a_{w}}{y_{w}}\right]_{R_{w}}$ lies in the kernel $L_{w}$ of $\delta_{w}$. Let us briefly recall the argument from 28] regarding how for a fixed $w$ this implies that $a_{w}$ lies in the tight closure of $\mathbf{x}_{w} R_{w}$. Namely, since the Frobenius $\mathbf{F}_{w}$ acts on the local cohomology groups, the kernel $L_{w}$ is invariant under its action by functoriality. Hence

$$
\mathbf{F}_{w}^{m}\left(\left[\frac{a_{w}}{y_{w}}\right]_{R_{w}}\right)=\left[\frac{\mathbf{F}_{w}^{m}\left(a_{w}\right)}{\mathbf{F}_{w}^{m}\left(y_{w}\right)}\right]_{R_{w}} \in L_{w} .
$$


Since $L_{w}$ is a proper subgroup of $\mathrm{H}_{\mathfrak{m}_{w}}^{d}\left(R_{w}\right)$ (note that $\delta_{w}^{d}$ is non-zero), the Matlis dual of $L_{w}$ is a proper homomorphic image of the canonical module $\omega_{R_{w}}$. Since the canonical module has rank one, the Matlis dual of $L_{w}$ has torsion, whence so does $L_{w}$ itself. Hence for some non-zero $c_{w} \in R_{w}$ we have $c_{w} L_{w}=0$. Together with (13), this yields

$$
\left[\frac{c_{w} \mathbf{F}_{w}^{m}\left(a_{w}\right)}{\mathbf{F}_{w}^{m}\left(y_{w}\right)}\right]_{R_{w}}=0
$$

for each $m$. Since almost each $R_{w}$ is Cohen-Macaulay, we get

$$
c_{w} \mathbf{F}_{w}^{m}\left(a_{w}\right) \in \mathbf{F}_{w}^{m}\left(\mathbf{x}_{w}\right) R_{w},
$$

for all $m$, proving our claim that $a_{w}$ lies in the tight closure of $\mathbf{x}_{w} R_{w}$. Since this holds for almost all $w$, we conclude that $a$ lies in the generic tight closure of $\mathbf{x} R$, which, by assumption, is just $\mathbf{x} R$. However, this means that $\left[\frac{a}{y}\right]_{R}$ is zero, contradiction.

Proof of Theorem $\mathrm{A}$. Since all properties localize, we may assume that $A$ and $B$ are moreover local and that $A \rightarrow B$ is a local homomorphism. Since $B$ is weakly generically F-regular by Theorem 3.3 , so is $A$, by Proposition 4.9, Therefore, $A$ is pseudo-rational by Theorem 6.2.

\section{UltRA-F-REgULAR RINGS AND LOG-TERMINAL SINGULARITIES}

In this section, we extend the argument from [27] in order to prove Theorem B]

7.1. $\mathbb{Q}$-Gorenstein singularities. Let $R$ be an equicharacteristic zero Noetherian local domain and put $X:=\operatorname{Spec} R$. We say that $R$ is $\mathbb{Q}$-Gorenstein if it is normal and some positive multiple of the canonical divisor $K_{X}$ is Cartier; the least such positive multiple is called the index of $R$. If $R$ is the homomorphic image of an excellent regular local ring (which is for instance the case if $R$ is complete), then $X$ admits an embedded resolution of singularities $f: Y \rightarrow X$ by [9]. If $E_{i}$ are the irreducible components of the exceptional locus of $f$, then the canonical divisor $K_{Y}$ is numerically equivalent to $f^{*}\left(K_{X}\right)+\sum a_{i} E_{i}$ (as $\mathbb{Q}$-divisors), for some $a_{i} \in \mathbb{Q}$. The rational number $a_{i}$ is called the discrepancy of $X$ along $E_{i}$; see 17, Definition 2.22]. If all $a_{i}>-1$, we call $R$ log-terminal (in case we only have a weak inequality, we call $R$ log-canonical).

7.2. Canonical cover. Recall the construction of the canonical cover of a $\mathbb{Q}$ Gorenstein local ring $R$ due to Kawamata. If $r$ is the index of $R$, then $\mathcal{O}_{X}\left(r K_{X}\right) \cong$ $\mathcal{O}_{X}$, where $X:=\operatorname{Spec} R$ and $K_{X}$ the canonical divisor of $X$. This isomorphism induces an $R$-algebra structure on

$$
\tilde{R}:=H^{0}\left(X, \mathcal{O}_{X} \oplus \mathcal{O}_{X}\left(K_{X}\right) \oplus \cdots \oplus \mathcal{O}_{X}\left((r-1) K_{X}\right)\right),
$$

which is called the canonical cover of $R$; see [16. An important property for our purposes is that $R \rightarrow \tilde{R}$ is étale in codimension one (see for instance [29, 4.12]). We also use the following result proven by Kawamata in [16, Proposition 1.7]:

7.3. Theorem. Let $R$ be a homomorphic image of an equicharacteristic zero, excellent regular local ring. If $R$ is $\mathbb{Q}$-Gorenstein, then it has log-terminal singularities if and only if its canonical cover is rational. 
7.4. Definition. Inspired by Kawamata's result, we can now give a resolution-free variant of log-terminal singularities. We call a Noetherian local domain pseudo-logterminal if it is $\mathbb{Q}$-Gorenstein and its canonical cover is pseudo-rational.

In the remainder of this section, $R$ is an equicharacteristic zero Noetherian local ring and $R_{w}$ is an approximation of $R$.

7.5. Ultra-F-regularity. We say that $R$ is ultra-F-regular, if it is a domain and for each non-zero $c \in R$, we can find an ultra-Frobenius $\mathbf{F}^{\varepsilon}$ such that the $R$-module morphism

$$
R \rightarrow{ }^{\varepsilon} \mathfrak{D}(R): x \mapsto c \mathbf{F}^{\varepsilon}(x)
$$

is pure. Note that in order for (14) to be $R$-linear, we need to view $\mathfrak{D}(R)$ as an $R$-algebra via $\mathbf{F}^{\varepsilon}$, that is to say, the target must be taken to be ${ }^{\varepsilon} \mathfrak{D}(R)$ (see $₫ 2.2$ ). Since $\mathfrak{D}(R)=\mathfrak{D}(\widehat{R})$, an analytically unramified local ring $R$ is ultra-F-regular if and only if its completion $\widehat{R}$ is.

Over normal domains, purity and cyclical purity are the same by $[10$, Theorem 2.6]. Hence for $R$ normal, the purity of (14) is equivalent to the weaker condition that for every $x \in R$ and every ideal $I \subseteq R$, we have

$$
c \mathbf{F}^{\varepsilon}(x) \in \mathbf{F}^{\varepsilon}(I) \mathfrak{D}(R) \quad \text { implies } \quad x \in I .
$$

One can show that if $R$ is moreover analytically unramified, then either condition entails normality, and hence in that case, they are equivalent (this follows for instance from the discussion below and the Briançon-Skoda property of generic tight closure).

7.6. Proposition. If $R$ is regular, then it is ultra-F-regular.

Proof. By the above discussion, we need only verify the weaker condition (15). In fact, we will show that for any $c$, we may take $\varepsilon=1$ in (15). Indeed, assume $c \mathbf{F}(x) \in \mathbf{F}(I) \mathfrak{D}(R)$. Since $\mathbf{F}$ preserves regular sequences, ${ }^{1} \mathfrak{D}(R)$ is a balanced big Cohen-Macaulay $R$-algebra whence is flat by [25, Theorem IV.1] or [12, Lemma 2.1(d)]. Hence

$$
c \in(\mathbf{F}(I) \mathfrak{D}(R): \mathbf{F}(x))=\mathbf{F}(I: x) \mathfrak{D}(R) .
$$

Suppose $x \notin I$. Since $(I: x)$ then lies in the maximal ideal of $R$, its image under $\mathbf{F}$ lies in the ideal of infinitesimals of $\mathfrak{D}(R)$. Hence $\mathbf{F}(I: x) \mathfrak{D}(R) \cap R=(0)$, contradicting that $c \neq 0$.

7.7. Theorem. If $R$ is analytically unramified and ultra-F-regular, then it is weakly generically F-regular, whence in particular pseudo-rational.

Proof. The last assertion follows from the first by Theorem 6.2. Since all properties are invariant under completion, we may assume that $R$ is complete. Let $I$ be an ideal in $R$ and $x \in \operatorname{cl}_{\text {gen }}(I)$. We want to show that $x \in I$. By [1, Proposition 6.24], there exists $c \in R$ such that almost all $c_{w}$ are test elements in $R_{w}$, where $c_{w}$ and $R_{w}$ are approximations of $c$ and $R$ respectively. Let $x_{w}$ and $I_{w}$ be approximations of $x$ and $I$ respectively, so that almost all $x_{w} \in I_{w}^{*}$. Hence, for almost all $w$ and all $e$, we have

$$
c_{w} \mathbf{F}_{w}^{e}\left(x_{w}\right) \in \mathbf{F}_{w}^{e}\left(I_{w}\right) R_{w} .
$$

By assumption, there is an ultra-Frobenius $\mathbf{F}^{\varepsilon}$ so that $x \mapsto c \mathbf{F}^{\varepsilon}(x)$ is pure whence cyclically pure, that is to say, so that (15) holds. Let $\varepsilon$ be the ultraproduct of 
integers $e_{w}$. Taking $e$ equal to $e_{w}$ in (16) and taking ultraproducts shows that $c \mathbf{F}^{\varepsilon}(x) \in \mathbf{F}^{\varepsilon}(I) \mathfrak{D}(R)$. Therefore, from (15) we get $x \in I$, as we wanted to show.

7.8. Proposition. Let $R \subseteq S$ be a finite extension of Noetherian local domains which is étale in codimension one. Let $c$ be a non-zero element of $R$ and $\mathbf{F}^{\varepsilon}$ an ultra-Frobenius. If $R \rightarrow{ }^{\varepsilon} \mathfrak{D}(R): x \mapsto c \mathbf{F}^{\varepsilon}(x)$ is pure, then so is its base change $S \rightarrow{ }^{\varepsilon} \mathfrak{D}(S): x \mapsto c \mathbf{F}^{\varepsilon}(x)$.

In particular, if $R$ is ultra-F-regular, then so is $S$.

Proof. Let $R \subseteq S$ be an arbitrary finite extension of $d$-dimensional Noetherian local domains and fix a non-zero element $c \in R$ and an ultra-Frobenius $\mathbf{F}^{\varepsilon}$. Let $\mathfrak{n}$ be the maximal ideal of $S$ and $\omega_{S}$ its canonical module. I claim that if $R \subseteq S$ is étale, then

$$
\varepsilon \mathfrak{D}(S) \cong S \otimes_{R} \varepsilon \mathfrak{D}(R) .
$$

Assuming the claim, let $R \subseteq S$ now only be étale in codimension one. It follows from the claim that the supports of the kernel and the cokernel of the natural map $S \otimes_{R}{ }^{\varepsilon} \mathfrak{D}(R) \rightarrow{ }^{\varepsilon} \mathfrak{D}(S)$ have codimension at least two. Hence the same is true for the base change

$$
\omega_{S} \otimes_{R} \varepsilon \mathfrak{D}(R) \rightarrow \omega_{S} \otimes_{S} \varepsilon \mathfrak{D}(S) .
$$

Applying the top local cohomology functor $\mathrm{H}_{\mathfrak{n}}^{d}$, we get from the long exact sequence of local cohomology and Grothendieck Vanishing, an isomorphism

$$
\mathrm{H}_{\mathfrak{n}}^{d}\left(\omega_{S} \otimes_{R} \varepsilon \mathfrak{D}(R)\right) \cong \mathrm{H}_{\mathfrak{n}}^{d}\left(\omega_{S} \otimes_{S} \varepsilon \mathfrak{D}(S)\right) .
$$

Recall that by Grothendieck duality, $\mathrm{H}_{\mathfrak{n}}^{d}\left(\omega_{S}\right)$ is the injective hull $E$ of the residue field of $S$.

Let $c_{\varepsilon, R}$ denote the $R$-linear morphism $R \rightarrow{ }^{\varepsilon} \mathfrak{D}(R): x \mapsto c \mathbf{F}^{\varepsilon}(x)$. For an arbitrary $R$-module $M$, let $c_{\varepsilon, R, M}: M \rightarrow M \otimes_{R} \varepsilon \mathfrak{D}(R)$ be the base change of $c_{\varepsilon, R}$ over $M$. In particular, we have a commutative diagram

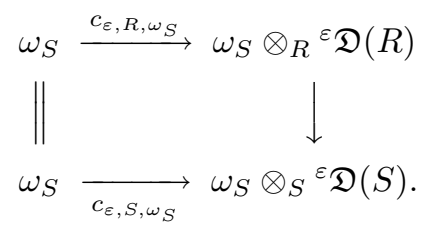

Taking top local cohomology yields the outer square in the following commutative diagram:

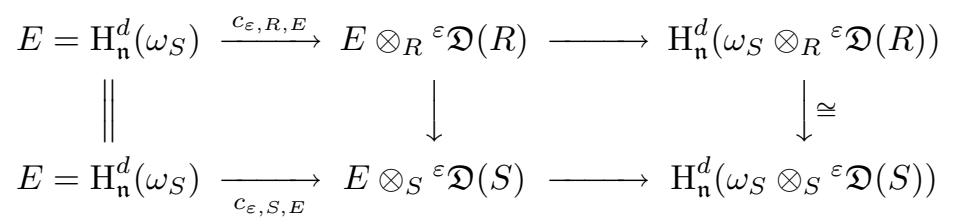

where the isomorphism at the right comes from (18). Since $c_{\varepsilon, R}$ is pure, so is its base change $c_{\varepsilon, R, \omega_{S}}$. Purity is preserved when taking cohomology, so that the top composite map in (19) is pure, whence so is the bottom composite map, since it is isomorphic to it. Since $c_{\varepsilon, S, E}$ is a factor of this map, it is itself pure, whence in particular injective. By [12, Lemma 2.1(e)], to verify the purity of $c_{\varepsilon, S}$, one only needs to show that its base change $c_{\varepsilon, S, E}$ over $E$ is injective, and this is exactly what we just showed. 
To prove the claim (17), observe that if $R \rightarrow S$ is étale with approximation $R_{w} \rightarrow S_{w}$, then almost all of these are étale. Indeed, by [20, Corollary 3.16], we can write $S$ as $R[X] / I$, with $X=\left(X_{1}, \ldots, X_{n}\right)$ and $I=\left(f_{1}, \ldots, f_{n}\right) R[X]$, such that the Jacobian $\mathrm{J}\left(f_{1}, \ldots, f_{n}\right)$ is a unit in $R$, and by Łos' Theorem, this property is preserved for almost all approximations. Quite generally, if $C \rightarrow D$ is an étale extension of characteristic $p$ domains, then we have for each $e$ an isomorphism ${ }^{e} D \cong D \otimes{ }_{C}{ }^{e} C$ (see for instance [15, p. 50] or the proof of [29, Theorem 4.15]). Applied to the current situation, we get ${ }^{e} S_{w} \cong S_{w} \otimes_{R_{w}}{ }^{e} R_{w}$ (see [15, p. 50]). Therefore, applied with $e=: e_{w}$, where $e_{w}$ is an approximation of $\varepsilon$, we get after taking ultraproducts,

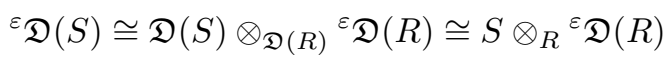

as required, where we used the isomorphism $\mathfrak{D}(S) \cong S \otimes_{R} \mathfrak{D}(R)$, which holds by [1, §4.10.4], since $R \rightarrow S$ is finite.

To prove the last assertion, we have to show that we can find for each non-zero $c \in S$ an ultra-Frobenius $\mathbf{F}^{\varepsilon}$ such that $c_{\varepsilon, S}$ is pure. However, if we can do this for some non-zero multiple of $c$, then we can also do this for $c$, and hence, since $S$ is finite over $R$, we may assume without loss of generality that $c \in R$. Since $R$ is ultraF-regular, we can therefore find an ultra-Frobenius $\mathbf{F}^{\varepsilon}$ such that $c_{\varepsilon, R}$ is pure, and hence by the first assertion, so then is $c_{\varepsilon, S}$, proving that $S$ is ultra-F-regular.

7.9. Proposition. Let $R \rightarrow S$ be a cyclically pure homomorphism of equicharacteristic zero Noetherian local rings. If $S$ is ultra-F-regular and analytically unramified, then so is $R$.

Proof. Since $\widehat{R} \rightarrow \widehat{S}$ is again cyclically pure by [1, Lemma 6.7], we may assume without loss of generality that $S$ is complete. Let $c \in R$ be non-zero and let $\mathbf{F}^{\varepsilon}$ be an ultra-Frobenius for which the $S$-module morphism

$$
c_{\varepsilon, S}: S \rightarrow{ }^{\varepsilon} \mathfrak{D}(S): x \mapsto c \mathbf{F}^{\varepsilon}(x)
$$

is pure. We want to show that the same is true upon replacing $S$ by $R$, that is to say, that $c_{\varepsilon, R}$ is pure. Since $S$ is weakly generically F-regular by Theorem 7.7, so is $R$ by Proposition 4.9. Hence $R$ is in particular normal by Theorem 4.3, so that it suffices to verify (15). Let $x \in R$ and $I \subseteq R$ be such that $c \mathbf{F}^{\varepsilon}(x) \in \mathbf{F}^{\varepsilon}(I) \mathfrak{D}(R)$. Therefore, $x$ belongs to $I S$ by (20), whence to $I S \cap R=I$ by cyclical purity.

Note that in the proof, the condition that $S$ is analytically unramified was only used to get the normality of $R$.

Proof of Theorem B. Proposition 7.6 yields that $S$ is ultra-F-regular, whence so is $R$ by Proposition 7.9, Let $\tilde{R}$ be the canonical cover of $R$. By Proposition 7.8, $\tilde{R}$ is also ultra-F-regular, whence pseudo-rational by Theorem 7.7 .

\section{REFERENCES}

[1] M. Aschenbrenner and H. Schoutens, Lefschetz extensions, tight closure and big CohenMacaulay algebras, Israel Journal of Mathematics (2007), to appear.

[2] J.-F. Boutot, Singularités rationelles et quotients par les groupes réductifs, Invent. Math. 88 (1987), 65-68. MR0877006 (88a:14005)

[3] W. Bruns and J. Herzog, Cohen-Macaulay rings, Cambridge University Press, Cambridge, 1993. MR1251956 (95h:13020)

[4] J. Eagon and M. Hochster, R-sequences and indeterminates, Quart. J. Math. Oxford Ser. 25 (1974), 61-71. MR0337934(49:2703) 
[5] P. Eklof, Ultraproducts for algebraists, Handbook of Mathematical Logic, North-Holland Publishing, 1977, pp. 105-137. MR0491125 (58:10395)

[6] N. Hara, A characterization of rational singularities in terms of injectivity of Frobenius maps, Amer. J. Math. 120 (1998), 981-996. MR.1646049 (99h:13005)

[7] R. Hartshorne, Local cohomology, Lect. Notes in Math., vol. 41, Springer-Verlag, 1967. MR0224620 (37:219)

[8] , Algebraic geometry, Springer-Verlag, New York, 1977. MR0463157 (57:3116)

[9] H. Hironaka, Resolution of singularities of an algebraic variety over a field of characteristic zero, Ann. of Math. 79 (1964), 109-326. MR0199184 (33:7333)

[10] M. Hochster, Cyclic purity versus purity in excellent Noetherian rings, Trans. Amer. Math. Soc. 231 (1977), 463-488. MR0463152 (57:3111)

[11] _ The tight integral closure of a set of ideals, J. Algebra 230 (2000), no. 1, 184-203. MR:1774763 (2002f:13009)

[12] M. Hochster and C. Huneke, Applications of the existence of big Cohen-Macaulay algebras, Adv. in Math. 113 (1995), 45-117. MR1332808 (96d:13014)

[13] M. Hochster and J. Roberts, Rings of invariants of reductive groups acting on regular rings are Cohen-Macaulay, Adv. in Math. 13 (1974), 115-175. MR0347810 (50:311)

[14] W. Hodges, Model theory, Cambridge University Press, Cambridge, 1993. MR 1221741 (94e:03002)

[15] C. Huneke, Tight closure and its applications, CBMS Regional Conf. Ser. in Math, vol. 88, Amer. Math. Soc., 1996. MR1377268 (96m:13001)

[16] Y. Kawamata, The cone of curves of algebraic varieties, Ann. of Math. 119 (1984), 603-633. MR0744865 (86c:14013b)

[17] J. Kollár and S. Mori, Birational geometry and algebraic varieties, Cambridge University Press, Cambridge, 1998. MR1658959 (2000b:14018)

[18] J. Lipman and B. Teissier, Pseudo-rational local rings and a theorem of Briançon-Skoda about integral closures of ideals, Michigan Math. J. 28 (1981), 97-116. MR0600418 (82f:14004)

[19] H. Matsumura, Commutative ring theory, Cambridge University Press, Cambridge, 1986. MR0879273 (88h:13001)

[20] J. Milne, Etale cohomology, 33, Princeton Math., 1980. MR0559531 (81j:14002)

[21] H. Schoutens, Existentially closed models of the theory of Artinian local rings, J. Symbolic Logic 64 (1999), 825-845. MR1777790 (2001g:03068)

[22] _ Lefschetz principle applied to symbolic powers, J. Algebra Appl. 2 (2003), 177-187. MR.1980407 (2004c:13040)

[23] , Non-standard tight closure for affine $\mathbb{C}$-algebras, Manuscripta Math. 111 (2003), 379-412. MR1993501 (2004m:13019)

[24] A non-standard proof of the Briançon-Skoda theorem, Proc. Amer. Math. Soc. 131 (2003), 103-112. MR1929029 (2003i:13004)

[25] _ Projective dimension and the singular locus, Comm. Algebra 31 (2003), 217-239. MR.1969220 (2005e:13020)

[26] Canonical big Cohen-Macaulay algebras and rational singularities, Illinois J. Math. 48 (2004), 131-150. MR2048219 (2005e:13012)

[27] _ Log-terminal singularities and vanishing theorems via non-standard tight closure, J. Alg. Geom. 14 (2005), 357-390. MR2123234 (2006e:13005)

[28] K. Smith, F-rational rings have rational singularities, Amer. J. Math. 119 (1997), 159-180. MR.1428062 (97k:13004)

[29] , Vanishing, singularities and effective bounds via prime characteristic local algebra, Algebraic geometry - Santa Cruz 1995, Proc. Sympos. Pure Math., vol. 62, Amer. Math. Soc., Providence, RI, 1997, pp. 289-325. MR.1492526 (99a:14026)

Department of Mathematics, City University of New York, 365 Fifth Avenue, New York, New York 10016

E-mail address: hschoutens@citytech.cuny.edu 